\title{
Características sociodemográficas e clínicas de idosos institucionalizados: contribuições para o cuidado de enfermagem
}

\author{
Sociodemographic and clinical characteristics of institutionalized older adults: contributions \\ to nursing care
}

\section{Características sociodemográficas y clínicas de ancianos institucionalizados: contribuciones al cuidado de enfermería}

\author{
Cintia Lira Borges ${ }^{I}$; Maria Josefina da Silva ${ }^{\text {II }}$; Jorge Wilker Bezerra Clares ${ }^{\text {III }}$; \\ Jéssica de Menezes Nogueira ${ }^{I V}$; Maria Célia de Freitas ${ }^{V}$
}

\begin{abstract}
RESUMO: Estudo transversal e descritivo que objetivou descrever as características sociodemográficas e clínicas de idosos institucionalizados. Participaram da pesquisa 54 idosos residentes numa instituição de longa permanência de Fortaleza/CE, mediante aplicação de formulário, em 2011. A população estudada apresentou idade média de $72,4 \pm 8,5$ anos, predominando os idosos do sexo masculino $(61,1 \%)$, solteiros $(46,3 \%)$, analfabetos $(46,3 \%)$, aposentados $(77,8 \%)$. Quanto à institucionalização, 51,9\% residiam na instituição há menos de cinco anos, 29,6\% eram ex-moradores de rua e 72,2\% não recebiam visitas. Quanto às características clínicas, 81 ,5\% possuíam alguma doença crônica, 83,3\% faziam uso contínuo de medicação; os níveis pressóricos e glicêmicos estiveram controlados para $57,4 \%$ e $81,1 \%$ idosos, respectivamente. Quanto ao perfil de fragilidade, $74,1 \%$ dos idosos encontravam-se frágeis. Esses dados podem subsidiar as ações dos enfermeiros na promoção de uma atenção mais efetiva e individualizada ao idoso institucionalizado.
\end{abstract}

Palavras-Chave: Idoso; instituição de longa permanência para idosos; características da população; enfermagem.

\begin{abstract}
This cross-cutting, descriptive study described the sociodemographic and clinical characteristics of institutionalized older adults. Fifty-four elderly residents at a long-stay institution in Fortaleza, Ceara State, Brazil, participated by responding to a form in 2011. Study population mean age was $72.4 \pm 8.5$ years; they were predominantly elderly males $(61.1 \%)$, single $(46.3 \%)$, illiterate $(46.3 \%)$ and retired $(77.8 \%)$. Institutionalization was expressed as: $51.9 \%$ had lived in the institution less than five years; $29.6 \%$ were former street dwellers; and $72.2 \%$ received no visits. Clinical characteristics included: $81.5 \%$ had a chronic disease; $83.3 \%$ made continuous use of medication; and blood pressure and blood glucose levels were controlled in $57.4 \%$ and $81.1 \%$ participants, respectively. Frailty profile was given as $74.1 \%$ of the elderly being fragile. These data can support the actions of nurses in promoting more effective and individualized care for institutionalized elderly.

Keywords: Elderly; homes for the elderly; population characteristics; nursing.
\end{abstract}

RESUMEN: Estudio transversal y descriptivo, cuyo objetivo fue describir las características sociodemográficas y clínicas de ancianos institucionalizados. Han participado de la encuesta 54 ancianos que viven en una institución de larga estancia de Fortaleza/Ceará, a quienes se les aplicó un formulario en 2011. La población estudiada tenía una edad media de 72,4 $\pm 8,5$ años, predominantemente varones ancianos $(61,1 \%)$, solteros $(46,3 \%)$, analfabetos $(46,3 \%)$, jubilados $(77,8 \%)$. En cuanto a la institucionalización, un $51,9 \%$ vivía en la institución desde hacía menos de cinco años, un 29,6\% había vivido en las calles y un 72,2\% no recibía visitas. Respecto a las características clínicas, un $81,5 \%$ tenía alguna enfermedad crónica, un $83,3 \%$ utilizaba continuadamente medicación; los niveles de presión arterial y glucosa fueron controlados en $57,4 \%$ y $81,1 \%$ de los participantes, respectivamente. En cuanto al perfil de fragilidad, de los ancianos, un $74,1 \%$ se mostraba frágil. Estos datos pueden apoyar las acciones de los enfermeros en el sentido de promover una atención más eficaz e individualizada a los ancianos institucionalizados

Palabras Clave: Anciano; hogares de larga estancia para ancianos; características de la población; enfermería.

\section{INTRODUÇÃO}

O envelhecimento populacional é um fenômeno acentuado no Brasil e no mundo, de grande impacto na estrutura econômica e sanitária das sociedades, tendo em vista o predomínio de doenças crônico-degenerativas entre os idosos, elevando a demanda por serviços de saúde e políticas públicas específicas para esse grupoํ․

'Enfermeira. Mestranda do Programa de Pós-Graduação Cuidados Clínicos em Enfermagem e Saúde da Universidade Estadual do Ceará. Integrante do Grupo de Pesquisa Enfermagem, Educação, Saúde e Sociedade. Fortaleza, Ceará, Brasil. E-mail: cintialiraborges@yahoo.com.br.

"Enfermeira. Doutora em Enfermagem. Professora Adjunta do Programa de Pós-Graduação em Enfermagem da Universidade Federal do Ceará. Fortaleza, Ceará, Brasil.E-mail: mjosefina@terra.com.br.

IIIEnfermeiro. Mestrando do Programa de Pós-Graduação Cuidados Clínicos em Enfermagem e Saúde da Universidade Estadual do Ceará. Integrante do Grupo de Pesquisa Enfermagem, Educação, Saúde e Sociedade. Fortaleza, Ceará, Brasil. E-mail: jorgewilker clares@yahoo.com.br.

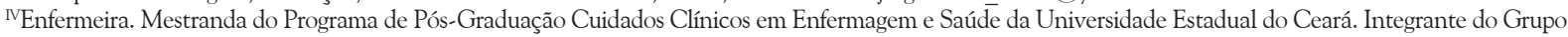
de Pesquisa Enfermagem, Educação, Saúde e Sociedade. Fortaleza, Ceará, Brasil. E-mail: jessicademenezesn@gmail.com.

vEnfermeira. Doutora em Enfermagem Fundamental. Professora Adjunta do Programa de Pós-Graduação Cuidados Clínicos em Enfermagem e Saúde da Universidade Estadual do Ceará. Integrante do Grupo de Pesquisa Enfermagem, Educação, Saúde e Sociedade. Fortaleza, Ceará, Brasil. E-mail: celfrei@ hotmail.com. 
Somam-se a isso as modificações contemporâneas nos arranjos domiciliares, contribuindo para a redução da disponibilidade de apoio e insuficiência familiar no cuidado aos idosos, o que tem acarretado o aumento da procura por instituições de longa permanência para idosos (ILPI) como alternativa de moradia e de cuidados permanentes a esse segmento etário da população ${ }^{2}$.

Contudo, essas instituições ainda representam um desafio, uma vez que as alterações próprias do envelhecimento e as doenças preexistentes podem ser agravadas pela institucionalização, gerando diferentes níveis de dependência no idoso. Destaca-se que, mesmo o idoso independente, quando institucionalizado, pode se tornar dependente em razão de dificuldades para aceitar e se adaptar às novas condições de vida, bem como à falta de motivação e de encorajamento comuns nesse ambiente ${ }^{3}$.

Conhecer as características da população a qual assiste é fundamental para o redirecionamento da atenção à saúde dos idosos institucionalizados, subsidia as ações de enfermagem, com base nas demandas específicas desse grupo, possibilitando desenvolver atividades de promoção da saúde e prevenção de doenças, com vistas a evitar a instalação de dependência e incapacidade funcional.

Diante de tais considerações, a investigação das características dos idosos institucionalizados justificase em virtude da necessidade de planejar e realizar atividades assistenciais e educativas no âmbito das ILPI, visando à prevenção de dependência física e mental nesse grupo. Sendo assim, o estudo objetivou descrever as características sociodemográficas e clínicas de idosos residentes em uma ILPI de Fortaleza/CE, Brasil.

\section{REVISÃo DE LITERATURA}

As ILPI são instituições governamentais ou não governamentais, de caráter residencial, destinadas tanto a idosos independentes em situação de carência de renda e/ou familiar, quanto a idosos dependentes, que necessitem de cuidados prolongados ${ }^{4}$.

Nos países europeus, verifica-se que cerca de 20\% da população idosa com alguma incapacidade funcional reside em instituições de longa permanência ${ }^{5,6}$. No Brasil, levantamento censitário realizado entre 2007 e 2009 verificou que 84 mil idosos residem nessas instituições, o que representa menos de $1 \%$ da população idosa do país ${ }^{4}$.

Pouca oferta de instituições, altos custos, preconceitos e estereótipos associados à preferência do cuidado aos idosos pelas famílias são alguns dos fatores que podem justificar a baixa proporção de idosos brasileiros residindo nas ILPI se comparado à realidade dos países desenvolvidos. Entretanto, acredita-se que a demanda por ILPI tende a crescer nos próximos anos, não só pelo envelhecimento populacional contemporâneo, mas também pelas mudanças nos arranjos familiares e pela situação de pobreza em que vive grande parte das famílias brasileiras?

Para o seu pleno funcionamento, essas instituições necessitam de uma equipe composta por enfermeiro, técnicos em enfermagem, médico, nutricionista, psicólogo, assistente social, fisioterapeuta, educador físico e cuidadores $^{8}$. O enfermeiro atuante em ILPI desenvolve um trabalho articulado com os demais integrantes da equipe multiprofissional de saúde, direcionado ao cuidado eficaz dos idosos residentes, mediante ações educativas, assistenciais, gerenciais e de pesquisa.

Ressalta-se que a atenção à saúde do idoso institucionalizado tem como finalidade principal a manutenção de um bom estado de saúde e a postergação da vida ativa nesse ambiente, com autonomia e independência física, psíquica e social. Portanto, participar ativamente do contexto social e manter-se com autonomia é essencial para as pessoas idosas, além de contribuir para a saúde e o bem-estar?.

Nesse sentido, atenção especial deve ser dada ao idoso institucionalizado, a fim de prevenir a incapacidade funcional. Para tal prevenção, é necessário que os enfermeiros conheçam as características desses idosos, sendo importante a realização de estudos de diagnóstico situacional, a fim de dispor de conhecimentos essenciais para melhorar a assistência ofertada no âmbito das ILPI por meio do planejamento de ações de impacto com enfoque nas demandas locais, resultando numa atuação mais efetiva e individualizada.

\section{Metodologia}

Estudo tipo descritivo, transversal, de abordagem quantitativa, realizado em uma ILPI pública do Município de Fortaleza/CE, integrada à Secretaria do Trabalho e Desenvolvimento Social, a qual congrega idosos abandonados, vítimas de violência ou em qualquer outra situação de vulnerabilidade social.

Para compor a amostra, foram utilizados os seguintes critérios de inclusão: ter 60 anos ou mais de idade, ser capaz de responder às perguntas e querer participar do estudo. Participaram da pesquisa 54 idosos, de ambos os sexos, em atendimento aos critérios de inclusão preestabelecidos. Ressalta-se a assinatura ou impressão digital no termo de consentimento livre e esclarecido por todos os participantes.

Os dados foram coletados durante o mês de março de 2011, após aprovação do projeto pelo Comitê de Ética em Pesquisa do Complexo Universitário da Universidade Federal do Ceará (protocolo 164/10).

A coleta foi realizada diariamente, nos períodos da manhã e da tarde, conforme a escala dos pesquisadores. Os dados foram obtidos mediante aplicação de formulários estruturados, em ambiente reservado na própria instituição, individualmente, com duração média de 20 minutos, além da busca de informações nos prontuários dos idosos. 
Para informações sobre o perfil sociodemográfico do idoso, as variáveis selecionadas foram: sexo, idade, escolaridade, estado civil, ocupação, tempo e motivos da institucionalização e suporte social. Para a caracterização clínica da amostra, foram coletadas variáveis antropométricas e fisiológicas, bioquímicas, doenças crônicas, uso de medicação e níveis de fragilidade.

Foram selecionadas as seguintes variáveis antropométricas e fisiológicas: peso, altura, índice de massa corporal (IMC), pressão arterial. A variável bioquímica analisada foi o nível glicêmico.

Para as variáveis IMC, glicemia e pressão arterial aplicaram-se os parâmetros estabelecidos no Caderno de Atenção Básica no 19 , do Ministério da Saúde ${ }^{10}$, a saber: IMC classificado em baixo peso $(\leq 22 \mathrm{~kg} / \mathrm{m} 2)$, peso adequado $(23-26 \mathrm{~kg} / \mathrm{m} 2)$ ou sobrepeso $(\geq 27 \mathrm{~kg} /$ $\mathrm{m} 2)$; níveis glicêmicos classificados em hipoglicemia ( $<80 \mathrm{mg} / \mathrm{dl})$, bom controle glicêmico $(80-159 \mathrm{mg} / \mathrm{dl})$, controle glicêmico aceitável (160-179 mg/dl) ou controle glicêmico ruim ( $\geq 180 \mathrm{mg} / \mathrm{dl})$; níveis pressóricos classificados em normal (<120 x $80 \mathrm{mmHg})$, pré-hipertensão (120-139 x 80-89 mmHg), hipertensão estágio I (140-159 x 90-99 mmHg) ou hipertensão estágio II ( $\geq 160$ x 100 $\mathrm{mmHg}$ ). Ressalta-se que, quando as pressões sistólica e diastólica se situam em categorias diferentes, deve se considerar o maior valor para classificação do estágio.

As informações sobre doenças crônicas e uso de medicamentos foram obtidas mediante a coleta nos registros dos prontuários.

O nível de fragilidade foi mensurado por meio da Edmonton Frail Scale (EFS) validada para a língua portuguesa e composta por nove domínios, distribuídos em 11 itens com pontuação de 0 a 17 pontos; categorizando em não fragilidade ( $0-4$ pontos), aparentemente vulnerável (5-6 pontos), fragilidade leve (7-8 pontos), fragilidade moderada (9-10 pontos) e fragilidade grave ( $\geq 10$ pontos). Quanto maior a pontuação, maior o nível de fragilidade ${ }^{11}$.

A coleta foi realizada em duas etapas. Primeiramente, houve o levantamento dos dados sociodemográficos e aplicação da EFS; em seguida, a avaliação antropométrica, fisiológica e bioquímica. Esta avaliação foi operacionalizada da seguinte forma: o peso corporal em quilogramas foi verificado com os idosos descalços em balança antropométrica (Britania ${ }^{\circledR}$ ), devidamente testada e calibrada, com precisão de $0,1 \mathrm{~kg}$, destinada exclusivamente para a pesquisa; a altura foi medida em metros com fita métrica inelástica, graduada em $0,5 \mathrm{~cm}$, com idoso descalço, os calcanhares juntos e olhar no horizonte; a glicemia capilar foi medida em mg/dl e verificada sem a obrigatoriedade do idoso estar em jejum, tendo sido utilizado o glicosímetro Accu-Check® Active; a pressão arterial foi aferida no braço direito, na posição sentada.

Os resultados foram processados e tabulados no programa Statistic Package for Social Science versão 19.0. Para tratamento dos dados, optou-se pelo uso da estatística descritiva, com números relativos e absolutos, sendo apresentados sob a forma de tabela.

\section{Resultados}

Participaram da pesquisa 54 idosos institucionalizados, que atenderam aos critérios de inclusão do estudo, sendo $33(61,1 \%)$ homens e 21 (38,9\%) mulheres. A idade variou de 60 a 90 anos, com média de 72,4 anos e desvio-padrão de 8,5 anos. No tangente à situação conjugal, verificou-se a predominância de solteiros (46,3\%), sendo inexistentes casos na situação de idosos que se declararam casados. A escolaridade mostrou-se baixa, predominando os analfabetos, 25 (46,3\%). No que se refere à ocupação, 12 (22,2\%) não eram aposentados, segundo a Tabela 1.

TABELA 1: Distribuição dos idosos institucionalizados, segundo características sociodemográficas. Fortaleza/CE, Brasil, 2011. $(\mathrm{N}=54)$

\begin{tabular}{|c|c|c|}
\hline Variáveis & $f$ & $\%$ \\
\hline \multicolumn{3}{|l|}{ Sexo } \\
\hline Masculino & 33 & 61,1 \\
\hline Feminino & 21 & 38,9 \\
\hline \multicolumn{3}{|l|}{ Idade } \\
\hline $60-69$ & 23 & 42,6 \\
\hline $70-79$ & 16 & 29,6 \\
\hline 80 ou mais & 15 & 27,8 \\
\hline \multicolumn{3}{|l|}{ Estado civil } \\
\hline Solteiro & 25 & 46,3 \\
\hline Separado/divorciado & 20 & 37,0 \\
\hline Viúvo & 9 & 16,7 \\
\hline \multicolumn{3}{|l|}{ Escolaridade } \\
\hline Analfabeto & 25 & 46,3 \\
\hline Alfabetizado & 11 & 20,4 \\
\hline Ensino fundamental incompleto & 12 & 22,2 \\
\hline Ensino fundamental completo & 2 & 3,7 \\
\hline Ensino médio incompleto & 2 & 3,7 \\
\hline Ensino médio completo & 2 & 3,7 \\
\hline \multicolumn{3}{|l|}{ Aposentado } \\
\hline Sim & 42 & 77,8 \\
\hline Não & 12 & 22,2 \\
\hline \multicolumn{3}{|l|}{ Tempo de institucionalização } \\
\hline Até 5 anos & 28 & 51,9 \\
\hline 6-9 anos & 13 & 24,1 \\
\hline 10 anos ou mais & 13 & 24,1 \\
\hline \multicolumn{3}{|l|}{ Motivo da institucionalização } \\
\hline Morador de rua & 16 & 29,6 \\
\hline Abandono/negligência & 10 & 18,5 \\
\hline Morava sozinho & 7 & 13,0 \\
\hline Violência & 5 & 9,3 \\
\hline Outros & 16 & 29,6 \\
\hline \multicolumn{3}{|l|}{ Recebe visitas } \\
\hline Sim & 15 & 27,8 \\
\hline Não & 39 & 72,2 \\
\hline \multicolumn{3}{|l|}{ Quem visita } \\
\hline Ninguém & 39 & 72,2 \\
\hline Amigos & 7 & 13,0 \\
\hline Filhos & 3 & 5,6 \\
\hline Outros & 5 & 9,2 \\
\hline
\end{tabular}


Em relação às características da institucionalização, 28 (51,9\%) idosos residiam na ILPI há menos de cinco anos. Os principais motivos apontados para a institucionalização foram: ser morador de rua, $16(29,6 \%)$ e abandono/negligência da família, 10 (18,6\%). Quanto à rede de suporte social anterior à institucionalização, 39 (72,2\%) idosos afirmaram não receber visitas externas, em detrimento a 15 (27,8\%) que recebem. Os amigos constituem os principais visitantes para 7 (13\%) idosos, conforme a Tabela 1.

As características clínicas dos idosos institucionalizados estão relacionadas na Tabela 2. Quanto ao IMC, observou-se que 12 (22,2\%) e 11 (20,4\%) idosos foram classificados como baixo peso e sobrepeso, respectivamente. A maioria dos idosos pesquisados $(81,5 \%)$ apresentou pelo menos uma doença crônica, com média de 1,28 doenças e desvio-padrão de 0,94 doenças por idoso. Identificou-se nos prontuários diversidade de doenças, sendo a hipertensão arterial $(45,5 \%)$ e o diabetes $(20,4 \%)$ as mais frequentes. Apesar da maior prevalência dessas doenças, tanto os níveis pressóricos quanto os glicêmicos estiveram controlados para 31 $(57,4 \%)$ e $43(81,1 \%)$ idosos, respectivamente.

TABELA 2: Distribuição dos idosos institucionalizados, segundo características clínicas. Fortaleza/CE, Brasil, 2011. ( $N=54)$

\begin{tabular}{|c|c|c|}
\hline Variáveis & $f$ & $\%$ \\
\hline \multicolumn{3}{|l|}{ Doença crônica } \\
\hline Sim & 44 & 81,5 \\
\hline Não & 10 & 18,5 \\
\hline \multicolumn{3}{|l|}{ Tipo de doença crônica } \\
\hline Hipertensão arterial & 20 & 45,5 \\
\hline Diabetes & 9 & 20,4 \\
\hline Cardiopatia & 3 & 6,8 \\
\hline Outras & 40 & 90,9 \\
\hline \multicolumn{3}{|l|}{ Necessidade de medicamentos } \\
\hline Sim & 45 & 83,3 \\
\hline Não & 9 & 16,7 \\
\hline \multicolumn{3}{|l|}{ IMC } \\
\hline Baixo peso & 12 & 22,2 \\
\hline Adequado & 31 & 57,4 \\
\hline Sobrepeso & 11 & 20,4 \\
\hline \multicolumn{3}{|l|}{ Níveis glicêmicos (mg/dl)* } \\
\hline$<80$ & 02 & 3,8 \\
\hline $80-160$ & 43 & 81,1 \\
\hline $161-$ & 2 & 3,8 \\
\hline 180 & 6 & 11,3 \\
\hline$>180$ & & \\
\hline \multicolumn{3}{|l|}{ Níveis pressóricos (mmHg) } \\
\hline$<120 \times 80$ & 31 & 57,4 \\
\hline $120-139 \times 80-89$ & 18 & 33,3 \\
\hline $140-159 \times 90-99$ & 3 & 5,6 \\
\hline$\geq 160 \times 100$ & 2 & 3,7 \\
\hline \multicolumn{3}{|l|}{ Fragilidade } \\
\hline Não apresenta & 2 & 3,7 \\
\hline Aparentemente vulnerável & 12 & 22,2 \\
\hline Fragilidade leve & 14 & 27,8 \\
\hline Fragilidade moderada & 15 & 25,9 \\
\hline Fragilidade severa & 11 & 20,4 \\
\hline
\end{tabular}

$(*)$ Houve uma recusa para a realização do teste glicêmico.
Quanto ao consumo de medicamentos, 45 $(83,3 \%)$ idosos necessitam do uso contínuo de medicação devido às morbidades existentes, dos quais 20 $(44,4 \%)$ fazem o uso de cinco a oito drogas diferentes, com maior prevalência para os psicotrópicos e os medicamentos clínicos, utilizados por 34 (63\%) idosos, cada. Em relação ao perfil de fragilidade, 40 (74,1\%) idosos estavam frágeis, variando de fragilidade leve a severa, e 12 (22,2\%) encontram-se aparentemente vulneráveis, segundo a Tabela 2 .

\section{Discussão}

Na pesquisa, predominaram os idosos do sexo masculino $(61,1 \%)$, fato também observado em estudo com idosos institucionalizados no sul do país ${ }^{12}$. Esses dados diferem de outras pesquisas nacionais ${ }^{13} \mathrm{e}$ internacionais ${ }^{14,15}$, que demonstram um predomínio do sexo feminino nesse tipo de estabelecimento. Essa divergência pode estar relacionada ao fato de a ILPI onde o presente estudo foi desenvolvido abrigar, na maioria dos casos, idosos moradores de rua, indigentes, perdidos, abandonados, sem referência familiar e social; no Brasil, essa situação é vivenciada predominantemente por homens ${ }^{16}$.

No que diz respeito ao nível instrucional dos idosos, verificou-se alta prevalência de pessoas com pouco ou nenhum grau de escolaridade. Tal achado já era esperado, uma vez que esses idosos viveram suas infâncias numa época em que a educação era pouco valorizada $^{17}$. Além disso, muitos eram moradores de rua e/ou provenientes de comunidades socialmente vulneráveis, com indicadores sociais e econômicos precários, justificando os baixos níveis de escolaridade.

Os aspectos socioeconômicos têm relação com a situação de saúde e o padrão de morbimortalidade da população e devem ser considerados durante o acompanhamento de saúde e planejamento de estratégias de cuidado pelos profissionais de saúde e, em particular, os enfermeiros, subsidiando ações de saúde direcionadas às necessidades do idoso institucionalizado.

Além disso, é possível inferir que as condições socioeconômicas desfavoráveis podem ter contribuído para a institucionalização desses idosos, uma vez que, atualmente, esse processo tem sido influenciado pelas mudanças nos arranjos familiares somados à insuficiência da família no cuidado aos seus idosos. Nessa diretiva, um estudo apontou que a presença de conflitos familiares, os problemas financeiros e não ter família constituíram os principais motivos para a institucionalização de sujeitos na faixa etária de 60 anos ou mais de idade ${ }^{18}$.

Os importantes quantitativos de analfabetos (46,3\%), de ex-moradores de rua $(29,6 \%)$ e de vítimas de abandono $(18,5 \%)$, além do número reduzido de visitas recebidas, denotam a frágil rede de apoio famil- 
iar e/ou social dos idosos estudados, sendo acentuada pela restrição da convivência social e pelo tempo de institucionalização.

As redes sociais de apoio são fundamentais para a melhoria da qualidade de vida dos idosos, possibilitando estabelecer relações sociais e fortalecer o uso de estratégias de enfrentamento para os desafios impostos pela velhice ${ }^{19}$. Dessa forma, o enfermeiro inserido nesse cenário tem participação fundamental na construção de uma estrutura de apoio ao idoso institucionalizado, devendo fornecer suporte social, emocional e afetivo, beneficiando a saúde e o bem-estar físico e mental dos sujeitos sob seus cuidados. Deve, ainda, sempre que possível, buscar estratégias para reintegrar esse idoso ao seu núcleo familiar, estimulando a responsabilização dos cuidados ao idoso pela família.

Sobre as características clínicas, verificou-se que a grande maioria dos idosos $(81,5 \%)$ apresentava pelo menos uma doença crônica, com maior destaque para as doenças cardiovasculares, dentre elas a hipertensão arterial e o diabetes. Esses achados assemelham-se aos de outros estudos realizados com idosos residentes em ILPI ${ }^{13}$.

As doenças cardiovasculares constituem condição de adoecimento elevado e comprometedor do envelhecimento da população, visto que, em certas situações, têm como resultado a perda da autonomia e/ ou independência dos idosos; além disso, representam a sua principal causa de óbito ${ }^{20}$. Destarte, a presença de hipertensão arterial e diabetes nesse grupo merece atenção, devido à maior vulnerabilidade frente às complicações cardio/cerebrovasculares determinadas pela associação entre as condições clínicas e outros fatores acumulados ao longo do tempo.

Apesar de a hipertensão arterial e o diabetes terem apresentado percentuais mais elevados nessa população, se comparadas às demais doenças referidas, verificou-se que os níveis pressóricos e glicêmicos, na maioria dos casos, estavam dentro dos limites considerados aceitáveis, o que pode ser resultado da utilização de medicações específicas para o controle dessas morbidades.

Ressalta-se que, na ILPI estudada, o controle da medicação é responsabilidade dos profissionais de enfermagem, mesmo dos idosos funcionalmente independentes, reforçando a necessidade de prevenir quadros de agudização e sequelas das doenças preexistentes, visto que elas podem resultar em incapacidades funcionais permanentes.

Verificou-se, ainda, o consumo de extensa quantidade de medicação pelos idosos em consonância ao relato de apresentarem morbidades: 45 (83,3\%) necessitam de medicação de uso contínuo, dos quais 20 $(44,4 \%)$ fazem uso de cinco a oito fármacos diferentes.

A prática da polifarmácia está associada ao aumento do risco e da gravidade das reações adversas, interações medicamentosas, toxicidade cumulativa, erros de medicação, redução da adesão ao tratamento e elevação da morbimortalidade. Nesse contexto, o enfermeiro deve estar atento para o uso racional de medicamentos pelos idosos, por meio do aprazamento criterioso dos horários da prescrição médica, de modo a evitar a administração simultânea de drogas que possam interagir entre si ou com a dieta. Ademais, é necessário monitorar os efeitos colaterais de fármacos relacionados a desfechos negativos, visando prevenir e minimizar os eventos adversos, contribuindo para o uso de maneira mais segura de medicamentos pelos idosos institucionalizados ${ }^{21}$.

O enfermeiro deve possuir conhecimento da farmacologia e ser capaz de detectar iatrogenias na população idosa. Além do aprazamento adequado das medicações, este profissional deve manter diálogo com o pessoal médico nas situações de necessidade de mudança da medicação, visto que o idoso pode apresentar efeitos colaterais ou reações adversas, sendo a observação da ocorrência desses efeitos indesejáveis uma das tarefas da enfermagem. Deve também desconfiar sempre da utilização da polifarmácia, realizando avaliação multidimensional do idoso. Dessa forma, é possível detectar necessidades/problemas de saúde que indiquem a real precisão da medicação; estar atento para os horários das alimentações e as interações com as medicações de uso crônico, bem como para a inclusão de medicamentos em aguda, como antibióticos e anti-inflamatórios e as possíveis interações.

Destaca-se a identificação, depois de detectados os diagnósticos de enfermagem, dos riscos que o fármaco pode estar causando ao organismo, como, por exemplo, um paciente com diagnóstico de enfermagem de constipação ter uma série de fatores farmacológicos relacionados que podem ser a causa desse problema. Portanto, é imprescindível que, durante a sistematização da assistência de enfermagem e o desenvolvimento da prática clínica, o enfermeiro perceba qual a função desses medicamentos os quais, com uso concomitante, possam estar contribuindo para a potencialização negativa do diagnóstico. Desse modo, esse profissional poderá interagir com o médico, organizando um plano de cuidados, no intuito de substituir ou evitar aquela medicação para a reestabilização do quadro clínico.

Atentando para o prejuízo do uso continuado de medicamentos e da polifarmácia para a qualidade de vida do idoso, o enfermeiro, durante sua prática clínica, pode desenvolver estratégias que viabilizem comportamentos de saúde e, possivelmente, a diminuição do uso exacerbado de medicamentos, com vistas ao bem-estar, qualidade de vida, autonomia e melhora do estado de saúde dos idosos institucionalizados.

$\mathrm{Na}$ avaliação do IMC, verificou-se frequência importante de idosos com desequilíbrio no estado nutricional, com percentuais de 22,2\% para baixo peso 
e 20,4\% para sobrepeso. Resultados semelhantes foram obtidos em trabalho realizado com 42 idosos institucionalizados no município de São Paulo, com 21,4\% dos idosos classificados como baixo peso e $33,4 \%$ com excesso de peso ${ }^{22}$.

Os problemas associados ao estado nutricional do idoso podem acelerar o aparecimento de fragilidades e vulnerabilidades, dificultando a recuperação dos idosos e reduzindo, significativamente, seu tempo de vida, principalmente na presença de doenças crônicas.

Assim, a avaliação do estado nutricional é uma importante ferramenta para o diagnóstico da saúde e nutrição, favorecendo orientações educativas e dietéticas, como também o monitoramento de intervenções terapêuticas em pessoas idosas ${ }^{23}$. Portanto, o estado nutricional, como elemento essencial para a avaliação de saúde do idoso, deve ser investigado durante a consulta de enfermagem, com o objetivo de prevenir a instalação de problemas decorrentes de carências nutricionais.

Quanto ao perfil de fragilidade, 74,1\% e 22,2\% dos idosos apresentaram-se frágeis e aparentemente vulneráveis, respectivamente. Esses dados corroboram os resultados de um estudo realizado com 100 idosos atendidos em um ambulatório rio-grandense-do-sul, que identificou a presença de algum grau de fragilidade em $84 \%$ da amostra ${ }^{24}$.

A fragilidade é definida como uma síndrome clínica caracterizada por diminuição de reserva e pela resistência reduzida aos estressores, resultante de declínio cumulativo nos sistemas fisiológicos (principalmente neuroendócrino, imunológico e musculoesquelético), causando vulnerabilidade às condições adversas entre os idosos. Dessa forma, é considerada como um significativo fator de risco para queda, incapacidade, hospitalização e morte entre idosos ${ }^{25}$.

Nesse contexto, reforça-se a importância da avaliação multidimensional do idoso, como possibilidade de detectar os fatores de risco para fragilidade e atuar de maneira preventiva no surgimento e/ou piora desse estado ${ }^{11}$. Ressalta-se, ainda, que o cuidado ao idoso fragilizado, bem como ao idoso vulnerável, seja realizado por uma equipe interdisciplinar, que deve estar preparada para identificar e intervir nos problemas de saúde associados à fragilidade no idoso, objetivando não somente a reabilitação, mas, principalmente, o estabelecimento de medidas preventivas e educativas para a saúde dos idosos institucionalizados ${ }^{24}$.

$\bigcirc$ profissional enfermeiro, se capacitado para realizar uma avaliação global do idoso, de forma multidimensional, pode prevenir o desenvolvimento ou agravamento da fragilidade, minimizando hospitalizações e taxas de morbimortalidade nesse segmento etário ${ }^{26,27}$.

Nessa perspectiva, os cuidados de enfermagem às pessoas idosas institucionalizadas, muitas vezes já fragilizadas ou em processo de fragilização, devem propiciar a autonomia e independência, o que poderá otimizar a disposição para a resiliência, contribuindo para que sejam ativas, capazes de enfrentar as adversidades na velhice. Ainda, os idosos institucionalizados também se deparam, além dos agravos físicos de saúde, com perdas de papéis familiares que interferem em suas dimensões social, mental e psíquica. Para isso, é possível elaborar plano de cuidados ou aplicar intervenções de enfermagem a partir do conhecimento prévio das características sociais e culturais dos idosos residentes em ILPI, reconhecendo suas especificidades, a fim de estimular sua independência, respeitando sua autonomia, sua história de vida, expectativas diante do processo de institucionalização, bem como suas limitações, e incentivar sua integração ao grupo, o que resultará em melhor qualidade de vida a esses sujeitos.

\section{Conclusão}

A análise dos resultados obtidos permitiu conhecer as características sociodemográficas e clínicas de idosos institucionalizados. Os achados chamam atenção para a necessidade de cuidados realizados por uma equipe multiprofissional, com destaque para o enfermeiro, que deve lançar mão do conhecimento acerca das características desses idosos para a promoção de um cuidado individualizado, mais efetivo e eficaz.

Importante destacar, como limitação do estudo, o fato de ter sido realizado apenas com os idosos funcionalmente independentes de uma única instituição, traduzindo uma realidade específica. Destarte, a metodologia utilizada pode ser replicada em outras realidades e a realização de estudos dessa natureza deve ser encorajada, incluindo-se os idosos com diferentes níveis de dependência, a fim de serem obtidas bases mais seguras e concretas para o planejamento de estratégias e o atendimento integral à saúde da população residente em ILPI.

Por fim, espera-se que tais achados possam subsidiar o planejamento de cuidados de enfermagem efetivos e individualizados aos idosos institucionalizados, com base em suas reais necessidades e nos fatores de risco à saúde, com vistas ao bem-estar físico, mental e social desse grupo.

\section{REFERÊNCIAS}

1.Veras R. Envelhecimento populacional contemporâneo: demandas, desafios e inovações. Rev Saude Publica. 2009; 43:548-54.

2.Creutzberg M, Gonçalves LHT, Sobottka EA, Ojeda BS. Long-term care institutions for elders and the health system. Rev Latino-Am Enfermagem. 2007;15:1144-9. 3.Araújo MOPH, Ceolim MF. Avaliação do grau de independência de idosos residentes em instituições de longa permanência. Rev esc enferm USP. 2007;41:378-85.

4.Camarano AA, Kanso S. As instituições de longa permanência para idosos no Brasil. Rev bras estud popul. 2010; $27: 232-5$ 
5.Demographic change in the euro area: projections and consequences. European Central Bank Monthly Bulletin. 2006;49-64.

6.Giannakouris K. Ageing characterises the demographic perspectives of the European societies. Eurostat Statistics in focus. 2008; 72:1-11.

7.Camarano AA, organizadora. Características das instituições de longa permanência para idosos: região Nordeste. Brasília (DF): IPEA; 2008.

8.Silva BT, Santos SSC. Cuidados aos idosos institucionalizados: opiniões do sujeito coletivo enfermeiro para 2026. Acta Paul Enferm. 2010;23:775-81.

9.Santos SSC, Silva BT, Barlem ELD, Lopes RS. The nurse role in the seniors' long permanence institution. Rev Enferm UFPE online. 2008 [cited 2012 Aug 03];2:291-9. Available from: http://www.ufpe.br/revistaenfermagem/index.php/revista/article/view/351/pdf_386 10.Ministério da Saúde $(\mathrm{Br})$. Secretaria de Atenção à Saúde. Departamento de Atenção Básica. Envelhecimento e saúde da pessoa idosa. Brasília (DF): Editora MS; 2006. 11.Fabrício-Wehbe SCC, Schiaveto FV, Vendrusculo TRP, Haas VJ, Dantas RAS, Rodrigues RAP. Crosscultural adaptation and validity of the Edmonton Frail Scale - EFS in a brazilian elderly sample. Rev Latino-Am Enfermagem. 2009;17:1043-9.

12.Carreira L, Botelho MR, Matos PCB, Torres MM, Salci MA. Prevalência de depressão em idosos institucionalizados. Rev enferm UERJ. 2011;19:268-73.

13.Aires M, Paz AA, Perosa CT. Situação de saúde e grau de dependência de pessoas idosas institucionalizadas. Rev Gaúcha Enferm. 2009;30:492-9.

14.Onder G, Carpenter I, Finne-Soveri H, Gindin J, Frijters D, Henrard JC et al. Assessment of nursing home residents in Europe: the Services and Health for Elderly in Long Term care (SHELTER) study. BMC Health Serv Res. 2012 [cited 2012 Aug 30];12:5. Available from: http://www.biomedcentral.com/1472-6963/12/5.

15.Torres Camacho MJ, Vázquez Perozo M, Parellada Sabaté A, González Acosta ME. Disfagia en ancianos que viven en residencias geriátricas de Barcelona. Gerokomos. 2011;22:20-4.
16.Fundação Instituto de Pesquisas Econômicas. Principais resultados do perfil socioeconômico da população de moradores de rua da área central da cidade de São Paulo. Relatório Executivo. São Paulo: FIPE; 2010.

17.Davim RMB, Torres GV, Dantas SMM, Lima VM. Estudo com idosos de instituições asilares no município de Natal/RN: características socioeconômicas e de saúde. Rev Latino-Am Enfermagem. 2004;12:518-24.

18.Rissardo LK, Furlan MCR, Grandizolli G, Marcon SS, Carreira L. Sentimentos de residir em uma instituição de longa permanência: percepção de idosos asilados. Rev enferm UERJ. 2012;20:380-5.

19.Nardi EFR, Oliveira MLF. Conhecendo o apoio social ao cuidador familiar do idoso independente. Rev Gaúcha Enferm. 2008;29:47-53.

20.Romero AD, Silva MJ, Silva ARV, Freitas RWJF, Damasceno MMC. Características de uma população de idosos hipertensos atendida numa unidade de saúde da família. Rev RENE. 2010;11:72-8.

21.Secoli SR. Polifarmácia: interações e reações adversas no uso e medicamentos por idosos. Rev Bras Enferm. 2010;63:136-40.

22.Lehn F, Coelho HDS, Garcia MT, Scabar LF. Estado nutricional de idosos em uma instituição de longa permanência. J Health Sci Inst. 2012;30:53-8.

23.Lacerda NC, Santos SSC. Avaliação nutricional de idosos: um estudo bibliográfico. Rev RENE. 2007;8:60-70. 24.Remor CB, Bós AJG, Werlang MC. Características relacionadas ao perfil de fragilidade no idoso. Sci Med. 2011;21:107-12.

25.Fried LP, Ferrucci L, Darer J, Williamson JD, Anderson G. Untangling the concepts of disability, frailty and comorbidity: implications for improved targeting and a care. J Gerontol Med Sci. 2004;59:255-63.

26.Macedo C, Gazzola JM, Najas M. Síndrome da fragilidade no idoso: importância da fisioterapia. Arq Bras Ciênc Saúde. 2008;33:177-84.

27.Pinto NBF, Vieira LB, Pereira FMV, Reis AMM, Cassiani SHB. Interações medicamentosas em prescrições de idosos hipertensos. Rev enferm UERJ. 2014; 22 : 785-91. 